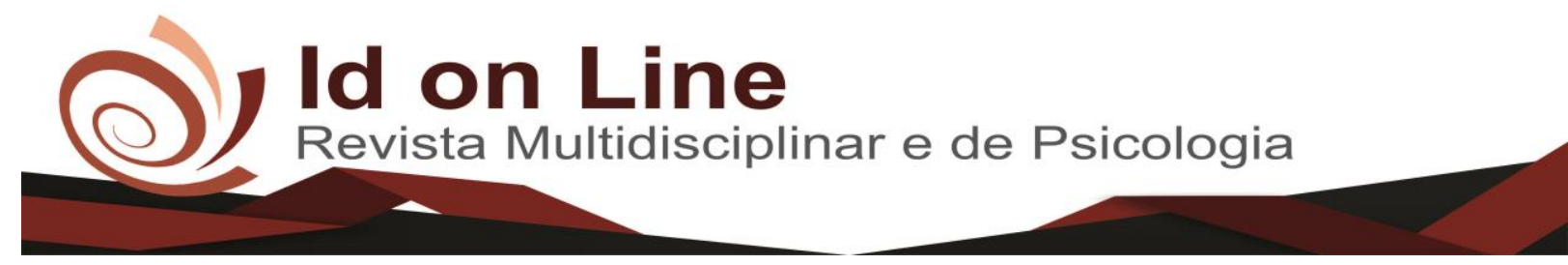

Artigo

\title{
Vínculo Profissional-Usuário na Estratégia Saúde da Família: Percepções de Idosos Hipertensos
}

\author{
Edglê Alves Ferreiral ; Déborah Cristina Silva Queiroz Alves²; Francisco José Braga Parnaíba ${ }^{3}$, \\ Ronnaby Vicente de Araújo ${ }^{4}$; Gesiany Pereira Vieira ${ }^{5}$; Amanda Pinheiro de Alencar ${ }^{6}$; \\ Thalita Franco Pinheiro Ferreira ${ }^{7}$; Rusy Maria Leite Amorim ${ }^{8}$
}

\begin{abstract}
Resumo: Objetivou-se identificar como se dá o nível de comunicação entre os pacientes idosos cadastrados no programa HIPERDIA com os profissionais da Estratégia Saúde da Família, quanto ao tratamento da Hipertensão Arterial Sistêmica. Tratou-se de um estudo exploratório, descritivo com abordagem qualitativa. O estudo foi desenvolvido com dez pacientes idosos hipertensos cadastrados no programa HIPERDIA vinculados a ESF Hermenegilda Paulino de Souza, onde as informações foram obtidas através de um grupo focal. Foi possível elencar a relação do nível de comunicação entre profissional e usuário em quatro categorias: vínculo deficiente; falta de vínculo com os profissionais; orientações adequadas no cuidado à saúde; e importância na tomada da medicação e suas complicações decorrentes. Torna-se imprescindível uma boa comunicação entre o profissional e o usuário na Estratégia Saúde da Família, assim, visando à redução de danos e complicações advindas da elevação dos níveis pressóricos da hipertensão arterial.
\end{abstract}

Palavras-chave: Vínculo, Estratégia Saúde da Família, Idosos, Hipertensão.

\section{Professional-User Bond in the Family Health Strategy: Perceptions of the Hypertensive Elderly}

\begin{abstract}
The objective of this study was to identify how the level of communication between the elderly patients registered in the HIPERDIA program with the professionals of the Family Health Strategy, regarding the treatment of Systemic Arterial Hypertension. It was an exploratory, descriptive study with a qualitative approach. The study was developed with ten hypertensive elderly patients enrolled in the HIPERDIA program linked to ESF Hermenegilda Paulino de Souza, where the information was obtained through a focus group. It was possible to list the level of communication between professional and user in four categories: poor link; lack of links with professionals; adequate guidelines for health care; and importance in taking the medication and its complications. It is essential to have good communication between the professional and the user in the Family Health Strategy, thus aiming at reducing the damages and complications caused by the elevation of the pressure levels of arterial hypertension.
\end{abstract}

Keywords: Bond, Family Health Strategy, Elderly, Hypertension.

\footnotetext{
${ }^{1}$ Residente em Saúde Coletiva pelo Programa de Residência Integrada em Saúde da Escola de Saúde Pública do Ceará - RISESP/CE. Graduado em Enfermagem pela Faculdade Vale do Salgado - FVS. E-mail: edgle.enf@ hotmail.com ${ }^{2}$ Hospital Geral Dr. César Cals. Graduada em Enfermagem pela Faculdade Vale do Salgado - FVS.

E-mail: enfa.deborahcristina@gmail.com

${ }^{3}$ Acadêmico em Enfermagem pela Universidade Regional do Cariri - URCA. E-mail: franciscobragaparnaiba@ gmail.com

${ }^{4}$ Acadêmico em Enfermagem pela Universidade Regional do Cariri - URCA. E-mail: ronnaby06@hotmail.com

${ }^{5}$ Acadêmica em Enfermagem pela Universidade Regional do Cariri - URCA. E-mail: gesianyvieira@ hotmail.com

${ }^{6}$ Acadêmica em Enfermagem pela Universidade Regional do Cariri - URCA. E-mail: alencarpinheiro1@ gmail.com

${ }^{7}$ Acadêmica em Enfermagem pela Universidade Regional do Cariri - URCA. E-mail: fthalita03@ gmail.com

${ }^{8}$ Acadêmica em Enfermagem pela Universidade Regional do Cariri - URCA. E-mail: rusy.leite @ hotmail.com
} 


\section{Introdução}

A Estratégia Saúde da Família (ESF) se configura como uma estratégia prioritária na expansão, consolidação e qualificação para a Atenção Básica (AB), na qual fortalece uma reorientação do processo de trabalho com grande potencial de ampliação e resolutividade, assim trazendo grandes impactos na situação de saúde do usuário e coletividades. Composta por uma equipe multiprofissional, formada por médico, enfermeiro, auxiliar e/ou técnico de enfermagem e Agente Comunitário de Saúde (ACS), podendo fazer parte da equipe o Agente de Combate a Endemias, cirurgião dentista e técnico ou auxiliar de saúde bucal (BRASIL, 2017).

Nesse contexto, prioriza-se as ações de prevenção, promoção e recuperação da saúde do usuário buscando de forma contínua a integralidade do cuidado. Assim, esses profissionais e a população adscrita criam vínculos de co-responsabilidade, o que facilita a identificação e o atendimento aos problemas de saúde da comunidade.

O vínculo apresenta-se articulado aos conceitos de humanização, responsabilização e da integralidade do cuidado, onde através do envolvimento entre os diferentes sujeitos envolvidos é capaz de fazer uma aproximação mútua entre estes indivíduos. Assim, o robustecimento do vínculo entre a equipe de saúde da família e o usuário torna-se de grande relevância, ao passo em que a ligação destes pontos levam ao favorecimento e a produção do cuidado mediante uma relação de confiança e partilha de comprometimento. (ILHA; DIAS; BACKES e BACKES, 2014)

Dentro desse contexto, ressalta-se que no cenário das políticas públicas de saúde no Brasil, o vínculo veio a surgir, mais especificamente, com a institucionalização do Sistema Único de Saúde (SUS) e o Programa Saúde da Família, mais tarde denominado ESF.

Dessa maneira, o vínculo constitui uma importante estratégia de cuidado da prática profissional no que tange a reorganização dos serviços assistências de saúde direcionados ao paciente, no sentido da garantia dos direitos do acesso universal, o atendimento humanizado, e a resolutividade dos serviços. Sob esse novo enfoque, o vínculo passa a ser considerado como um marco de destaque e grande relevância no SUS, através da mudança conceitual da relação entre o profissional e o usuário de um serviço de saúde (BACKES, et al., 2015). 
Em se tratando dos pacientes idosos hipertensos, cabe destacar que estes merecem especial atenção, pois a Hipertensão Arterial Sistêmica (HAS) apresenta alta prevalência, baixas taxas de controle e elevado número de abandono ao tratamento. Em decorrência a elevação da Pressão Arterial (PA), a HAS é considerada um fator desencadeante a outras patologias, bem como o infarto agudo do miocárdio, Acidente Vascular Encefálico (AVE), insuficiência renal crônica, e insuficiência cardíaca, principalmente, e quando associada a outros fatores de risco, assim como o diabetes melitus, sedentarismo, obesidade e tabagismo, os níveis pressóricos podem ainda serem mais elevados e ocasionar lesões de outros órgãos alvo (SILVA, 2015).

A idade avançada configura-se o principal fator de risco da HAS, principalmente indivíduos a partir dos 50 anos de idade, havendo maior prevalência entre os indivíduos do gênero masculino, porém com prevalência parecida entre ambos. Outros fatores de risco aparecem o excesso de peso, sedentarismo, ingesta aumentada de sal nas refeições, uso de álcool, além dos fatores socioeconômicos e genéticos (CARVALHO et al., 2013).

Sua prevalência no Brasil varia de $22 \%$ a $44 \%$, estimando em cerca de $32 \%$ em média, sendo que este número aumenta consideravelmente em relação a idade, chegando a mais de 50\% nos indivíduos com idade de 60 a 69 anos e $75 \%$ para indivíduos com idade superior a 70 anos. Entre os gêneros, a sua prevalência apresenta 35,8\% em homens e 30\% em mulheres (BRASIL, 2010).

Em 2001, aproximadamente 7,6 milhões de mortes no mundo foram atribuídas pelo aumento progressivo dos níveis pressóricos, a partir de 115/75 mmHg de maneira linear, contínua e independente, sendo que 54\% dos óbitos em indivíduos com AVE, foram decorrentes de problemas associados a elevação da PA e ainda cerca de $47 \%$ dos óbitos por doença isquêmica do coração, se deram por meio de seu aumento progressivo, sendo a maioria em países de baixo e médio desenvolvimento econômico e maior parte em indivíduos com idade de 45 a 69 anos.

Nesse sentido há a necessidade de ações de promoção da saúde que visem à prevenção e o diagnóstico precoce, mediante a efetivação de uma equipe multiprofissional, promovendo uma assistência qualificada, para que possa contribuir de maneira eficaz no retardamento das complicações (SANTOS; NERY; MATUMOTO, 2013).

Torna-se ponderoso, isto posto, ampliar os debates acerca do vínculo como uma ferramenta de tecnologia leve, apto de promover o viver saudável dos indivíduos idosos 
hipertensos, além da família e comunidade. Nesse aspecto, o vínculo se revela como um elemento ao mesmo tempo facilitador e desafiador na ESF, tornando-se necessária a ampliação das discussões para a resolução da lacuna no conhecimento acerca da importância do mesmo para o desenvolvimento e a reorganização das práticas profissionais visando à centralização do cuidado no usuário, a partir da ótica dos próprios profissionais.

O presente estudo teve como objetivo identificar como se dá o nível de comunicação entre os pacientes idosos cadastrados no programa HIPERDIA (cadastramento e acompanhamento de pacientes Hipertensos e Diabéticos) com os profissionais da ESF, quanto ao tratamento da HAS.

\section{Metodologia}

Trata-se de um estudo do tipo exploratório, descritivo com abordagem qualitativa. O estudo foi desenvolvido com pacientes idosos hipertensos cadastrados no programa HIPERDIA do Ministério da Saúde, vinculados à Estratégia Saúde da Família Hermenegilda Paulino de Sousa, situado no Município de Umarí, Estado do Ceará.

As informações foram obtidas por meio da técnica do Grupo Focal (GF), caracterizada por sessões previamente planejadas no compartilhamento de momentos específicos com os usuários através de reuniões e discussões em grupo, onde foram discutidos assuntos pertinentes aos interesses tanto do pesquisador quanto dos participantes (WIGGINS, 2004).

Foram convidados para participarem da pesquisa dez clientes idosos que detinham como característica especificadora serem idosos cadastrados no programa HIPERDIA. Os critérios de inclusão utilizados foram os seguintes: estarem na faixa etária a partir dos 60 anos de idade, residir dentro da área adscrita da ESF Hermenegilda Paulino de Sousa, concordar em participar da pesquisa, através da leitura do Termo de Consentimento Livre e Esclarecido e assinatura do Termo de Consentimento Pós-Esclarecido. Os critérios de exclusão utilizados foram os seguintes: aqueles idosos que não puderam se deslocar ou tivessem alguma deficiência senil, na qual impossibilitaria de participarem do grupo focal.

Os dados foram coletados em agosto de 2017, onde foi possível identificar os sujeitos por intermedeio das ACS e convidados por elas e pelo pesquisador durante uma 
visita em domicilio dos mesmos para participarem do GF, onde foi direcionado o local, data e horário para a realização da atividade proposta.

Foi realizado apenas uma sessão focal, visto que só há necessidade de outros encontros, quando ficam questões ou assuntos pendentes, não esclarecidos ou de acordo com o objetivo do estudo (BARBOUR, 2009).

Os participantes foram postos em um semicírculo ao redor do moderador, contando ainda com um observador que esteve na parte externa do semicírculo, para que fosse realizado as anotações precisas durante o momento e ainda uma anotadora (CAMPOS, 2005). Durante a seção, as falas dos integrantes da pesquisa foram gravadas como forma de garantia e fidedignidade das respostas no debate e posteriormente estas foram transcritas visando a garantia do sigilo e do anonimato. Para essa garantia, foi utilizado códigos, identificados pela letra I (idosos) e enumerados de acordo com a sequência de colocação das cadeiras no local e subsequente os arquivos foram salvos numa pasta renomeada de GF (grupo focal).

Após a discussão do grupo focal os dados foram transcritos, com a finalidade de facilitar a análise e discussão dos resultados. Para iniciar as discussões, inicialmente foram atribuídas Ideias Centrais (IC) e em seguida, os relatos foram organizados de acordo o Discurso do Sujeito Coletivo (DSC).

Lefevre, Lefevre (2014) apontam o DSC como uma técnica metodológica utilizada para organizar dados qualitativos, sendo fundamentada na teoria da representação social. Assim, torna-se um discurso-síntese organizado para discursos de sentido semelhante, por meio de procedimentos sistemáticos e padronizados.

Posteriormente, as falas foram analisadas mediante literatura pertinente. Após a transcrição integral do material coletado foi realizado uma leitura fluente das falas para a garantia de filtração do conteúdo, o que possibilitou o desenvolvimento de unidades de registro que deu origem as fontes de discussão do estudo.

A pesquisa foi desenvolvida obedecendo as recomendações da Resolução ${ }^{\circ} 466$ de 12 de dezembro de 2012, do Conselho Nacional de Saúde do Ministério da Saúde (BRASIL, 2013). 


\section{Resultados}

O grupo focal foi composto por um número de dez indivíduos, estes sendo idosos hipertensos cadastrados no programa HIPERDIA do Ministério da Saúde adscritos na ESF Hermenegilda Paulino de Sousa.

Ressalta-se que, dentre os indivíduos que compuseram o GF houve uma paridade em relação ao sexo, apontando cinco (50\%) do sexo masculino e outros cinco (50\%) do sexo feminino. A idade também apontou paridade em relação as faixas etárias, na qual compreendia dois (20\%) de 60 a 64 anos, dois (20\%) com idade de 65 a 69 anos, dois (20\%) de idade de 70 a 74 anos, outros dois (20\%) com idade de 75 a 79 anos de idade, e mais outros dois (20\%) com idade superior a 80 anos. Em se tratando do estado civil, notou-se uma prevalência de indivíduos casados, representando seis (60\%), dois (20\%) eram divorciados, e outros dois (20\%) eram viúvos. Em relação à escolaridade, três (30\%) eram analfabetos, cinco (50\%) possuíam o ensino fundamental incompleto, um (10\%) possuía o ensino fundamental completo e apenas uma pessoa (10\%) possuía o ensino médio completo. Em se tratando da renda familiar, três $(30 \%)$ dos participantes, tinham uma renda de até um salário mínimo e os outros sete $(70 \%)$ participantes possuem renda familiar de um entre dois salários mínimos.

Foi indagado aos participantes da pesquisa, uma questão cuja respostas foram transcritas, analisadas e organizadas em expressões chaves e ideias centrais, com posterior agrupamento no discurso do sujeito coletivo. Após análise das falas, foi possível identificar de forma mais concreta o nível de comunicação e vínculo entre profissional e os usuários, em relação às medidas de controle e tratamento da HAS, através do acompanhamento dos profissionais da unidade de saúde sobre os referidos pacientes.

Foi possível elencar a relação do nível de comunicação entre profissional e o paciente, após respostas dos integrantes quanto ao nível de comunicação e interação dos mesmos com os profissionais da Estratégia Saúde da Família, quando questionados se recebiam algum esclarecimento dos profissionais à importância na utilização correta da medicação, bem como um cuidado ao todo, no tratamento anti-hipertensivo, assim, as respostas foram agrupadas em quatro categorias e seus discursos respectivos a seguir: 


\section{IC I / DSC I - Vínculo deficiente}

"Nunca falaram não, porque quando eu ia, eu ia buscar o remédio e voltava para trás."

\section{IC II / DSC II - Falta de vínculo com os profissionais}

"Eu pra falar a verdade num conheço nem esse pessoal, num procurei nem a conhecer, você veja o tanto que eu sou errado, que num procurei nem a conhecer. Me ligo pouco."

\section{IC III / DSC III - Orientações adequadas no cuidado à saúde}

"Eles dizem que é bom tomar esse remédio, ter cuidado e que se não tiver lá, comprar né, e não deixe de tomar."

\section{IC IV / DSC IV - Importância na tomada da medicação e as complicações decorrentes}

“Elas falam pelo amor de Deus, não deixa de tomar o remédio da pressão, que já por duas vezes que eu fui parar no hospital, já fui duas vezes no hospital, quase morro, mas é descuido meu."

\section{Discussão}

Diante da análise de fala do personagem no DSC-1 vê-se uma grande falha do cliente ao procurar a unidade de saúde, notando-se um vínculo deficiente entre o profissional e o paciente, sendo possível identificar a falta de interesse em utilizar dos recursos disponíveis na Estratégia Saúde da Família, pois conforme sua fala ainda observa-se que quando comparece à unidade é apenas para buscar a sua medicação. 
Em consonância com Malta, Morais Neto e Silva Junior (2011) a falta de interesse dos pacientes idosos em aderirem ao SUS pelo nível primário de saúde é bastante baixa, pois grande parte deste público não leva sua doença a sério. Por se tratar de uma doença crônica, apontam os grandes riscos que estes estão sujeitos, levando a uma maior demanda às instituições hospitalares quando submetidos às complicações decorrentes.

Os fatos apresentados chegam a condizer um impacto cada vez mais crescente no sistema de saúde, em decorrência de complicações e nos altos custos representados pelas internações, onde poderiam ser tratados de maneiras eficazes, como na utilização adequada do tratamento, tanto no que se refere ao tratamento com agentes anti-hipertensivos quanto ao tratamento não medicamentoso.

Essa problemática é foco de discussão na literatura existente, onde é verificado por Brasil (2011) que a equipe de saúde da ESF tem atuação importante sobre medidas preventivas, que visem à redução de danos à população, cabendo ainda à equipe realizar a busca dos pacientes faltosos para a reinserção na unidade, dando assim a continuidade do seu tratamento anti-hipertensivo.

Ainda em concordância com o autor supracitado acima, a adesão a tratamentos por doenças crônicas na ESF é bastante decepcionante, muitas vezes, levando este fato a uma imensa preocupação, contudo reconhece a importância de ações e intervenções que buscam a incrementar a adesão destes pacientes e como resultados, dar a continuidade do tratamento e a redução das complicações, principalmente as demais doenças do aparelho circulatório, sendo por uso irregular da medicação ou no abandono do mesmo.

No caso da fala em DSC-2 observa-se a um fato mais preocupante quando o mesmo foi questionado sobre orientações e esclarecimento dos profissionais acerca da importância do tratamento anti-hipertensivo, o mesmo afirma que desconhece os profissionais, observando a total falta de vínculo entre profissional e usuário, ainda é possível perceber que o mesmo assume a culpa em não ter o interesse em procurar o serviço de saúde, não dando a importância necessária à sua saúde.

O resultado evidencia que a ida dos clientes na ESF é um fator de grande influência no que diz respeito ao seu tratamento, pois estes podem frequentar a unidade mensalmente, através das consultas programadas e/ou agendadas, conforme estabelece o Programa do HIPERDIA do Ministério da Saúde, onde são orientados pelo médico ou enfermeiro. Por outro lado a não ida por partes deste público pode levar a sérios problemas de saúde, uma 
vez que os mesmos são portadores de uma doença crônica, que, no entanto não existe cura, porém existe o tratamento.

Tal contexto vem a concordar com Santos, Nery e Matumoto (2013) na qual é constatado o acompanhamento dos pacientes idosos com HAS por profissionais de saúde, pois esta conduta se faz necessário na medida em que a idade avançada passa a apresentar maiores riscos em relação aos de menor idade.

Todavia, ressalta-se que o comparecimento às consultas pelos idosos de maneira regular leva o indivíduo ao acompanhamento adequado da HAS, tornando-se um fator positivo na adesão ao tratamento pelo monitoramento durante as consultas, com a finalidade de esclarecimento sobre a importância deste acompanhamento, e pela apresentação de melhores medidas a serem seguidas pelos idosos.

Analisando a fala do integrante em DSC-3, observa-se o relato do cliente pelos profissionais da unidade sobre a importância em utilizar os medicamentos antihipertensivos, orientando a comprá-los quando estes estiverem em falta no serviço de saúde.

Esses relatos se assemelham aos achados da pesquisa de Souza et al., (2014) onde foi relatado a importância em ações educativas, ao passo em que estas podem levar a formação com grupos de pacientes e familiares como uma estratégia de adequação destes pacientes na unidade, levando a construção de conhecimentos compartilhados aos demais membros, possibilitando a troca de informações e por permitir o apoio mútuo. Em relação a essas atividades, o paciente identifica-se com outros presentes com problemas semelhantes e assim formando o compartilhamento de experiências, discutindo reais soluções que possam servir de forma adequada dos problemas de saúde semelhantes aos seus.

Destarte, os benefícios da utilização da medicação anti-hipertensiva são imensos, principalmente na diminuição das complicações cardiovasculares. Diante este fato, tornase imprescindível a abordagem de ações educativas e terapêuticas em saúde.

Ressalta-se a importância da $\mathrm{AB}$ como mediadora na reinserção destes pacientes, cabendo aos profissionais à busca por meio da realização de ações de promoção em saúde, na prevenção e assistência do acompanhamento de forma longitudinal aos portadores de doenças crônicas e desta maneira criando vínculos diretos e responsabilizando-se pelos usuários adscritos na área (BRASIL, 2011). 
Em DSC-4, nota-se grande preocupação por parte dos profissionais e que orientam no cuidado intensivo sobre a medicação. Acredita-se que pelo motivo destes terem conhecimento que o cliente já veio a apresentar problemas advindos da elevação da pressão arterial, assim como constatado pelo personagem em sua fala.

Ao examinar a fala, percebe-se que a equipe de saúde da ESF apresentam uma importância fundamental no que se diz respeito ao processo de intervenções, no controle de doenças e de seus fatores de risco modificáveis. Entrando neste ponto, a educação em saúde passa como uma medida que levam o usuário as ações preventivas, por intermédio do processo educativo em saúde, e em relação a esses pacientes vem a ser considerada a chave para o sucesso do trabalho.

Cabe dizer que os profissionais de saúde da $\mathrm{AB}$, devem levar à comunidade uma grande representação em relação ao tratamento de doenças, bem como da HAS, pois estes atuam na detecção precoce, e por intermédio da aferição periódica da PA, incentivam sobre os cuidados e o respectivo controle de seus fatores de risco por meio de ações de educação em saúde e atuando ainda na administração direta dos serviços de saúde da unidade, no caso dos controles das consultas agendadas e ainda na busca dos pacientes faltosos, desta maneira o paciente sente-se muito mais valorizado, levando a sua responsabilização como seu verdadeiro instrumento de seu tratamento (DOURADO et al., 2011).

Em consonância com Carvalho, Almeida e Garbinato (2012), ainda complementam que os profissionais da unidade atuam nesse contexto com a finalidade não apenas em influenciar o comportamento do paciente para a obtenção de mudanças, mas sim na manutenção destas. É preciso usar a informação dentro de um processo educativo que leve as transformações, inclusive comportamentais. Vários instrumentos podem ser usados para incentivar o hipertenso, como no caso de caminhadas, jogos educativos, formação de grupos, utilização de materiais educativos, dentre outras medidas.

Logo é essencial que a educação em saúde é extremamente importante, como medidas voltadas aos idosos com o objetivo de orientarem quanto a ações de promoção da saúde, destacando a importância do tratamento específico de doenças crônicas, e por se tratar de um público bastante frágil destaca-se uma maior abordagem aos mesmos de forma sucinta. 
Destarte a participação ativa do cliente é consideravelmente importante no controle da doença e na prevenção de suas complicações, devendo haver engajamento suficiente tanto do paciente quanto por parte dos profissionais de saúde da unidade.

\section{Considerações Finais}

Ao longo da pesquisa foi possível identificar vários aspectos que estão diretamente relacionados com o nível de comunicação entre os profissionais de saúde da ESF com o usuário da respectiva área adscrita, assim compreendendo a utilização de vínculos por estes, que são considerados de extrema importância.

Através dos achados na pesquisa é possível evidenciar os principais aspectos direcionados na reação do vínculo entre profissional e paciente, onde foi possível identificar que os pacientes, em sua parte não têm o hábito de frequentarem a ESF, levando a uma falta ou até mesmo a inexistência de vínculos destes, e que nem sempre recebem algum incentivo sobre a importância na continuidade do seu tratamento anti-hipertensivo. Por outro lado, demais pacientes apresentam-se mais assíduos na unidade de saúde e que apresentam uma boa interação entre profissional e paciente.

No entanto, em se tratando do cliente idoso hipertenso, torna-se imprescindível uma boa comunicação entre o profissional e o usuário na ESF, pois não é possível obter o controle adequado do tratamento anti-hipertensivo da hipertensão arterial, se o paciente não foi instruído sobre os princípios no qual se fundamentam a importância do tratamento.

Como contribuição, o estudo propõe a inserção de medidas educativas que levem estes a compreenderem a importância na terapia medicamentosa, na utilização de uma busca ativa destes pacientes à unidade para realizarem suas consultas de maneira assídua, levando o indivíduo ao acompanhamento adequado visando assim à redução de danos e complicações advindas da elevação dos níveis pressóricos da hipertensão arterial.

Pode-se perceber que os pacientes do estudo necessitam de uma atenção dos profissionais da $\mathrm{AB}$ por ser constatado em parcela dos usuários do estudo, que o seu predomínio na unidade é frequentemente baixa. Dessa feita, o comprometimento em tal ação levará o indivíduo a ter uma maior assiduidade na unidade ocasionando menores 
complicações, menos ida a hospitais em decorrência de agravos e favorecendo melhor qualidade de vida.

Diante fatos apresentados, essa pesquisa mostrou-se relevante na medida em que buscou preencher com informações diversas lacunas existentes sobre o assunto. Espera-se que esse estudo venha a contribuir de maneira significativa à melhoria da qualidade de vida dos idosos hipertensos, servindo-se de subsidio em medidas e melhores estratégias que possam vincular o indivíduo idoso com HAS na ESF.

\section{Referências}

BACKES, D. S.; PEREIRA, A. D.; MARCHIORI, M. T.; RUPOLO, I.; BACKES, M. T. S.; BUSCHER, A. Vínculo profissional usuário: competência para a atuação na Estratégia Saúde da Família. Av Enfrm. Florianópolis. v. 33, n. 2, p. 222-229, 2015.

BARBOUR, R. Grupos focais. Porto Alegre: Artmed, 2009.

BRASIL. Conselho Nacional de Saúde. Resolução no 466, de 12 de dezembro de 2012. Aprova as normas regulamentadoras de pesquisas envolvendo seres humanos. Diário Oficial da República Federativa do Brasil, Brasília: Ministério da Saúde, n. 12, Seção 1, p. 59-59. 2013.

- Ministério da Saúde. Doenças crônicas não transmissíveis: estratégias de controle e desafios para os sistemas de saúde. $1^{\text {a }}$ ed. Brasília: Ministério da Saúde. 2011.

Ministério da Saúde. VI Diretrizes Brasileiras de Hipertensão. Brasília: Ministério da Saúde. 2010.

. Ministério da Saúde. Portaria no 2.436 de 21 de setembro de 2017. Diário Oficial da República Federativa do Brasil. Brasília: Ministério da Saúde. 2017.

CAMPOS, R. O. Pesquisa qualitativa em políticas, planejamento e gestão em saúde coletiva. Pesquisa qualitativa em saúde: múltiplos olhares. Campinas: Editora Unicamp, 2005 .

CARVAlHO, M. C.; AlMEIDA, A. P. M. de; GARBINATO, L. R. A assistência farmacêutica no atendimento aos pacientes do Hiperdia do ESF 18 e 19 da cidade de Dourados/MS. Interbio. Dourados. v. 6 n. 2 p. 5-15, 2012.

CARVALHO, M. V. de.; SIQUEIRA, L. B.; SOUSA, A. L. L.; JARDIM, P. C. B. V. A influência da hipertensão arterial na qualidade de vida. Arq Bras Cardiol. Goiânia. v. 100, n. 2. p. 164-174, 2013. 
DOURADO, C. S.; COSTA, K. N. de F. M.; OLIVEIRA, J. dos S.; LEADEBAL, O. D. C. P.; SILVA, G. R. F. da. Adesão ao tratamento de idosos com hipertensão em uma Unidade Básica de Saúde de João Pessoa, Estado da Paraíba. Acta Scientiarum Health Sciences. Maringá, v. 33 n. 1. p. 9-17, 2011.

ILHA, B; DIAS, M. V.; BACKES, D. S.; BACKES, M. T. S. Vínculo profissional-usuário em uma equipe da Estratégia Saúde da família. Cienc Cuid Saude. Rio Grande. v. 13, n. 3, p. 556-562, 2014.

LEFEVRE, F.; LEFEVRE, A. M. C. Discurso do sujeito coletivo que fala representações sociais e intervenções comunicativas. Texto Contexto Enferm. Florianópolis. v 23, n. 2, Abr-Jun, 2014.

MALTA, D. C.; MORAIS NETO, O. L. de; SILVA JUNIOR, J. B. da. Apresentação do plano de ações estratégicas para o enfrentamento das doenças crônicas não transmissíveis no Brasil, 2011 a 2022. Epidemiol. Serv. Saúde, Brasília, v. 20, n. 4, p. 425-438, 2011.

SANTOS, F. P. dos A.; NERY, A. A.; MATUMOTO, S. A produção do cuidado a usuárias com hipertensão arterial e as tecnologias em saúde. Rev Esc Enferm USP. São Paulo. v. 47, n. 1, p. 107-114, 2013.

SILVA, L. S. A. Hipertensão arterial sistêmica e morbidade cardiovascular: foco na epidemiologia no Brasil. Revista Hipertensão. Salvador. v. 17, n. 3, p. 163-168, 2015.

SOUZA, C. S de.; STEIN, A. T.; BASTOS, G. A. N.; PELLANDA, L. C. Controle da pressão arterial em hipertensos do Programa Hiperdia: Estudo de base territorial. Arq Bras Cardiol, Porto Alegre, v. 102, n. 8, p. 571-578, 2014.

WIGGINS, G. S. The analysis of focus groups in published research articles. The Canadian Journal of Program Evaluation. v. 19, n. 2, p. 143-164, 2004.

\section{Como citar este artigo (Formato ABNT):}

FERREIRA, Edglê Alves; ALVES, Déborah Cristina Silva Queiroz; PARNAÍBA, Francisco José Braga; ARAÚJO, Ronnaby Vicente de; VIEIRA, Gesiany Pereira; ALENCAR, Amanda Pinheiro de; FERREIRA, Thalita Franco Pinheiro; AMORIM, Rusy Maria Leite. Vínculo Profissional-Usuário na Estratégia Saúde da Família: Percepções de Idosos Hipertensos. Id on Line Rev.Mult. Psic., 2019, vol.13, n.43, p. 748-760. ISSN: 1981-1179.

Recebido: $11 / 12 / 2018$

Aceito: $12 / 12 / 2018$ 